\title{
La Migración y su Influencia Académica en los Estudiantes de la Carrera de Ingeniería Mecánica de la Espoch, Ecuador
}

\author{
Ginno Sidney Jarrín Zambrano, \\ Master en Gerencia Educativa y Especialista en Gestión Educativa, \\ Docente de la Escuela Superior Politécnica de Chimborazo, Ecuador \\ Jaqueline Elizabeth Balseca Castro, \\ Magister en Pequeñas y Medianas Empresas Mención Finanzas \\ Docente de la Escuela Superior Politécnica de Chimborazo, Ecuador \\ Marco Altamirano Balseca, \\ Magister en Gerencia de la Educación Abierta, \\ Docente de la Escuela Superior Politécnica de Chimborazo, Ecuador \\ Angélica María Paredes, \\ Colaboradora en la Investigación de Campo
}

Doi:10.19044/esj.2019.v15n19p41 ～URL:http://dx.doi.org/10.19044/esj.2019.v15n19p41

\section{Resumen}

El presente artículo se centra en el contexto dado por el alto número de estudiantes reprobados en la Escuela de Ingeniería Mecánica del tercer semestre de la ESPOCH en las diferentes áreas de estudio, un hecho que denota problemas de índole psicológico, social y económico, dado por un alto índice de población emigrante de las diferentes provincias del Ecuador. Con el fin de comprender esta situación la investigación se enmarcó dentro un campo de estudio de fácil comprensión y fácil reproducción de técnicas metodológicas y procedimientos donde se estudiaron diferentes muestras y una estructura de trabajo que abarca la recopilación de información y su contraste con las investigaciones realizadas. La investigación ratificó y comprobó la importancia que tienen los diferentes factores como el ambiente social, la psicología y el nivel de adaptación de los estudiantes sobre la capacidad y desempeño estudiantil de los mismos, frente al impacto que provoca el salir de la ciudad de residencia habitual de los estudiantes. El universo de estudio se enmarcó en los estudiantes de la carrera de Ingeniería Mecánica de la Escuela Superior Politécnica de Chimborazo. Se presentan los resultados de las entrevistas realizadas y a su vez las posibles conclusiones y recomendaciones hacia los docentes para contrarrestar este tipo de problemáticas sociales. 
Palabras claves: Migración, estudiantes, universidades, rendimiento académico

\title{
Migration and its Academic Influence on the Students of the Mechanical Engineering Career of Espoch, Ecuador
}

\section{Ginno Sidney Jarrín Zambrano,}

Master en Gerencia Educativa y Especialista en Gestión Educativa, Docente de la Escuela Superior Politécnica de Chimborazo, Ecuador

Jaqueline Elizabeth Balseca Castro,

Magister en Pequeñas y Medianas Empresas Mención Finanzas

Docente de la Escuela Superior Politécnica de Chimborazo, Ecuador

Marco Altamirano Balseca, Magister en Gerencia de la Educación Abierta,

Docente de la Escuela Superior Politécnica de Chimborazo, Ecuador

\section{Angélica María Paredes,}

Colaboradora en la Investigación de Campo

\begin{abstract}
This paper focuses on the context given by the high number of failed students in the School of Mechanical Engineering of the third semester of ESPOCH in the different areas of specialization. This problem was attributed to the psychological, social, and economic nature of a high index of emigrant population of the different provinces of Ecuador. In order to understand this situation, the research was framed within a field of study for easy understanding and reproduction of methodological techniques and procedures. Here, different samples were studied and a work structure that covers the collection of information and its contrast with the investigations was carried out. The research confirmed and verified the importance of the different factors such as the social environment, psychology, and the level of adaptation of the students based on the student's capacity and performance. Thus, this is in view of the impact caused by leaving the city where the students reside. The study framework is the career of Mechanical Engineering students of the Polytechnic School of Chimborazo. The results of the interviews conducted
\end{abstract}


are presented, and possible conclusions and recommendations to teachers were given to counteract this type of social problems.

Keywords: Migration, students, universities, academic performance.

\section{Introducción}

Con el paso de los años, Ecuador ha cambiado su sistema de educación. Se han hecho reformas educativas en cuanto a la educación superior para tener universidades de calidad en la mayor cantidad de ciudades que sean posibles, incrementando las oportunidades de estudio para los jóvenes del país.

Aunque parece un gran avance educativo, para la mayoría de los jóvenes no es suficiente y siguen optando por migrar a las grandes ciudades en busca de prestigiosas Universidades que puedan cumplir con las expectativas de sus padres y principalmente con los gustos y afinidades educativas que poseen.

Para entender por qué migran los estudiantes de unas ciudades a otras en busca de mejor calidad de estudio primero debemos entender que es la migración y lo haremos por su concepto general que es:

"La migración es el traslado o desplazamiento de la población de una región a otra o de un país a otro, con el consiguiente cambio de residencia; dicho movimiento constituye un fenómeno geográfico de relevante importancia en el mundo." (Núñez, Chavarría \& Molina, 2011). Entendiendo este concepto, es posible deducir que las personas que migran, en este caso los estudiantes, deben encontrar un nuevo estilo de vida y adaptarse a él, ya que conlleva al cambio de residencia y alejarse de su lugar de origen y de sus familiares, a los cuales no podrán acudir tal cual lo hacían en su lugar de origen, lo cual llevará a cabo una independencia social El alto número de los estudiantes reprobados en la Escuela de Ingeniería Mecánica del tercer semestre de la ESPOCH en las diferentes áreas de estudio denota problemas de índole psicológico, social y económico, dado por un alto índice de población emigrante de las diferentes provincias del Ecuador. El hecho de ser migrante hace que los niveles de asimilación y expresión de sentimientos disminuyan afectando su entorno tanto laboral como emocional.

En este contexto, con el presente artículo se busca comprender por qué los estudiantes eligen cambiar su lugar de residencia y migrar a otras ciudades donde haya universidades para el estudio de la educación superior. Se pudo identificar que no simplemente lo hacen por gusto o por capricho, aunque la migración es temporal hablando de los años que conlleva la obtención de un título de una carrera universitaria, sino que buscan la obtención de grados y poder seguir una formación profesional escogida por prestigio de la universidad o por diversas razones que conllevan al cambio total del estilo de vida. 


\section{Problema: Desarrollo Hipótesis}

La migración puede ser una de las principales causas de la pérdida de semestres y el retiro de los estudiantes de la carrera de Ingeniería Mecánica de la ESPOCH.

\section{Objetivo general}

Identificar la influencia de la migración en los estudiantes del tercer nivel de la carrera

de Ingeniería Mecánica de la ESPOCH mediante la recopilación cuantitativa del primery segundo parcial para determinar posibles causas del mal desemp eño estudiantil.

\section{Objetivos específicos}

- Valorar el estado psico socio afectivo de los estudiantes del tercer nivel de 1 a carrera de Ingeniería Mecánica de la ESPOCH aplicando una encuesta.

- Determinar la situación actual de los estudiantes del tercer nivel de la carrer a deIngeniería Mecánica de la ESPOCH como método regulador de baja aca démica.

- Proponer pausas socioafectivas en la ejecución de cada asignatura como ap oyo motivacional para lograr un aumento progresivo en el rendimiento académico.

\section{Metodología}

\section{Diseño de la investigación}

La investigación fue realizada en los estudiantes del tercer nivel de Ingeniería Mecánica de la ESPOCH, quienes han emigrado de las diferentes provincias del país; para ello se recopila la información de libros revistas, sitios web donde se determinan las causas y efectos del estudio de campo, gracias a que se recolectan los datos que caracterizan a dicha población. El estudio investigativo tiene un tiempo determinado deabril a agosto 2018.

\section{Nivel de investigación}

El nivel de investigación permite identificar las causas del bajo rendimiento académico plasmado en las notas del primer y segundo parcial descriptivo. Gracias a la obtención de los datos se pueden exponer las causasde la migración y esto a su vez el bajo rendimiento académico. 


\section{Descripción del ámbito de la investigación}

Población DIANA

La población con la cual se trabajó pertenece a la Facultad de Mecánica, Escuela de Ingeniería Mecánica de la ESPOCH, con un total de 20 estudiantes.

\section{Población experimental o de referencia}

Se aplicaron encuestas a los estudiantes del tercer nivel de la Escuela de Ingeniería Mecánica de la ESPOCH paralelo "A", en donde se analizan los datos obtenidos en la población migratoria, sus motivos y la influencia que presenta en el rendimiento académico.

\section{Actitud explicativa}

La población fue seleccionada para la investigación ya que presenta condiciones comunes del medio de procedencia para continuar con sus estudios, razón por la cual se alejan de la familia resultando afectado su campo psico socio afectivo y por ende el rendimiento académico.

\section{Actitud pragmática}

La población fue seleccionada para la investigación ya que presenta condiciones comunes del medio de procedencia para continuar con sus estudios, razón por la cual se alejan de la familia resultando afectado su campo psico socio afectivo y por ende el rendimiento académico.

\section{Técnicas e instrumentos}

\section{La observación}

La técnica que se empleará en la investigación es la observación, con la cual se evidencia el comportamiento además de la expresión corporal y familiar de la población requerida indagando para obtener resultados para el estudio investigativo.

\section{La encuesta}

Con el desarrollo de una encuesta con 11 ítems aplicada a la población a estudiar se recolectaron los aspectos primordiales para la comparación de la población residente - población emigrante.

\section{Operacionalización de variables}

Variable dependiente

Rendimiento académico en los estudiantes de tercer nivel de Ingeniería Mecánica de la ESPOCH 


\begin{tabular}{|c|c|c|c|}
\hline Conceptualizar & $\begin{array}{l}\text { Categorías o } \\
\text { dimensiones }\end{array}$ & Indicadores & Ítems Básicos \\
\hline $\begin{array}{l}\text { El rendimiento } \\
\text { académico es la } \\
\text { evaluación del } \\
\text { conocimiento } \\
\text { adquirido en el } \\
\text { ámbito educativo. } \\
\text { Es una medida de } \\
\text { las capacidades } \\
\text { del estudiante } \\
\text { que expresa lo } \\
\text { que ha aprendido a } \\
\text { lo largi del } \\
\text { proceso formativo. }\end{array}$ & $\begin{array}{l}\text {-Evaluación del } \\
\text { conocimiento } \\
\text {-Capacidades del } \\
\text { Estudiante que } \\
\text { expresa lo que ha } \\
\text { aprendido a lo largo } \\
\text { del proceso } \\
\text { formativo }\end{array}$ & $\begin{array}{l}\text { - Revisar los } \\
\text { temas tratados en } \\
\text { clase. } \\
\text { - Analizar diferentes } \\
\text { fuentes. } \\
\text { - Realizar ejercicios } \\
\text { de refuerzo. } \\
\text { - Pedir tutorías al } \\
\text { profesor o a otros } \\
\text { estudiantes. }\end{array}$ & $\begin{array}{l}\text { ¿Cómo Evalúa sus } \\
\text { conocimientos } \\
\text { adquiridos? } \\
\text { ¿Qué factores } \\
\text { complican su } \\
\text { buen rendimiento } \\
\text { académico? }\end{array}$ \\
\hline
\end{tabular}

Tabla 1. Detalle de la variable dependiente

Variable independiente: Migración de los estudiantes de tercer nivel de la Escuela de Ingeniería Mecánica de la ESPOCH:

\begin{tabular}{|c|c|c|c|}
\hline Conceptualizar & $\begin{array}{l}\text { Categorías o } \\
\text { dimensiones }\end{array}$ & Indicadores & Ítems Básicos \\
\hline $\begin{array}{l}\text { La migración de los } \\
\text { estudiantes es el } \\
\text { movimiento de } \\
\text { estos, consiste en } \\
\text { abandonar el lugar } \\
\text { de residencia para } \\
\text { establecerse en otra } \\
\text { región, } \\
\text { generalmente por } \\
\text { causas económicas, } \\
\text { sociales. }\end{array}$ & $\begin{array}{l}\text { - Abandonar el } \\
\text { Lugar de } \\
\text { residencia } \\
\text { para } \\
\text { establecerse } \\
\text { en otra región. } \\
\text { - Mejor oferta } \\
\text { académica. }\end{array}$ & $\begin{array}{l}\text { - Alejarse de la } \\
\text { familia. } \\
\text { - Adaptarse a un } \\
\text { nuevo estilo de } \\
\text { vida. } \\
\text { - Adquirir más } \\
\text { independencia. }\end{array}$ & $\begin{array}{l}\text { - Puntualizar } \\
\text { los motivos de } \\
\text { migración en } \\
\text { los estudiantes. } \\
\text { - Determinar el } \\
\text { factor que } \\
\text { interfiere en el } \\
\text { desempeño } \\
\text { de los estudiantes. }\end{array}$ \\
\hline
\end{tabular}

Tabla 2. Detalle de la variable independiente

\section{Análisis e interpretación de los resultados}

La encuesta realizada consta de 11 preguntas que abarcaron los aspectos más importantes de la influencia de la migración en los estudiantes del tercer nivel de la carrera de Ingeniería Mecánica de la ESPOCH. De 20 estudiantes encuestados, 17 fueron de sexo masculino y 3 de sexo femenino entre los 19 y 23 años, estableciéndose que un $40 \%$ son residentes y el restante $60 \%$ son migrantes.

\section{Provincias de origen}

La mayor cantidad de estudiantes migrantes provienen de la provincia de Tungurahua, teniendo un porcentaje total de 33.33\%; a continuación, está 
la provincia de Sucumbíos con un $25 \%$ y el resto de los alumnos proviene de distintas provincias en un número no muy elevado.

\section{Pregunta 1}

Señale los motivos por los que usted decidió estudiar en Riobamba

Los principales motivos por los que los estudiantes deciden estudiar en la escuela de Ingeniería Mecánica de la ESPOCH son especialmente debido al prestigio que posee la institución y que es una carrera de su agrado. Las respuestas en esta pregunta pueden haber sido una o varias, dependiendo de la persona encuestada.

\section{Pregunta 2}

¿Cuántos años ha estado viviendo en Riobamba?

El promedio aproximado de años que nuestra población ha estado viviendo en Riobamba es de 2.5 años, siendo 1.5 años el menor tiempo y 3 años el mayor. Uno de los estudiantes encuestados respondió que no vive en Riobamba, pero viaja todos los días, factor que se debe tomar en cuenta debido a que esto resulta importante en el desempeño de dicho alumno. De los estudiantes encuestados ese es el único caso, pero si se toma en cuenta a toda la escuela de Ingeniería Mecánica existirán muchos otros estudiantes que como él viajan todos los días, cosa que complica su rendimiento académico debido a la pérdida de tiempo en el viaje.

\section{Pregunta 3}

¿Vive actualmente con alguien?

De los 12 estudiantes migrantes encuestados la mitad vive con alguien, en general con un amigo o amigos, y la otra mitad viven solos; lo que nos permite suponer que la proporción de alumnos migrantes que viven acompañados es igual a los que viven solos.

\section{Pregunta 4}

¿Cuánto dinero dispone semanalmente para sus gastos?, ¿Es suficiente?

Se planteó si el dinero del que disponen los estudiantes a la semana es suficiente para sus gastos, a lo que el $100 \%$ de los encuestados respondió que subsisten con menos de 50\$; para 10 personas esa cantidad es suficiente, para dos no lo es.

\section{Pregunta 5}

¿Qué es lo más complicado para usted al ser migrante (comida, soledad, etc.)?

La principal complicación que tienen los alumnos migrantes es lo concerniente a sus estudios. Más del $40 \%$ del total de la población manifestó 
que tienen muchos inconvenientes al estudiar y realizar sus libres académicas, el $13.33 \%$ que dijo que la distancia a sus hogares los complica. La motivación es otro factor mencionado por el $13.33 \%$ de la población, al igual que los quehaceres del hogar, haciendo referencia más que todo a la comida, pues extrañan que al llegar de clases no esté alguien preparando el almuerzo o la cena. Al restante $20 \%$ le cuesta adaptarse al nuevo estilo de vida.

\section{Pregunta 6}

¿Cuántas horas al día están destinadas para sus estudios?

El promedio de horas extras que estos estudiantes dedican a sus estudios varía en un rango de 1 a 5 horas al día.

\section{Pregunta 7}

¿Cuál es la materia más complicada para usted en este semestre?

Más de la mitad de la población encuestada manifestó que tiene problemas con Análisis Matemático II, un 58.33\% del total; Química es la asignatura que le sigue, con un $16.67 \%$ de estudiantes del total que tiene problemas en esta cátedra; Física y Dibujo Mecánico comparten su porcentaje, siendo este de $8.33 \%$ del total.

\section{Pregunta 8}

¿Cuántas inasistencias a clases tiene a la semana?

La mayor parte de los estudiantes encuestados no tiene muchas inasistencias a clase, siendo 0 la cantidad de clases perdidas por la mayoría.

\section{Pregunta 9}

Se le preguntó también a los estudiantes como consideran la educación de la ESPOCH, resultados que en su mayoría son satisfactorios pues el $100 \%$ de ellos opinó que la calidad de la educación es excelente o buena, siendo 7 personas las que dijeron que es excelente y 5 que es buena.

\section{Pregunta 10}

¿Se considera una persona responsable tanto en su vida personal como estudiantil?

La pregunta es de carácter emocional, se refiere a si la persona se considera una responsable tanto en su vida personal como estudiantil. En esta pregunta 11 estudiantes respondieron que sí y tan solo 1 de ellos respondió que no.

\section{Pregunta 11}

Se cuestiona si la población considera que la migración influye directamente en su rendimiento académico. Resultados que se muestran en el gráfico. 


\section{Verificación de la hipótesis}

Se pudo determinar mediante la investigación que el cambio de región de estilo de vida y comportamiento afecta directamente en los estudiantes provenientes de otras provincias.

El principal problema que se presenta actualmente en estudiantes provenientes de ciudades alejadas a la universidad es la adaptación a una vida independiente, una vida más llena de responsabilidades e independencia personal.

Se comprobó en la investigación que la migración afecta directamente el rendimiento académico, ocasionando que los estudiantes tengan un menor rendimiento que el de los estudiantes residentes.

\section{Discusión}

En un primer trabajo correspondiente a la Red Europea de Migración (2015), quienes realizaron "Migración de los Estudiantes Internacionales", se referencian debido a la comprensibilidad de todo su cuerpo y desarrollo el cual trata y da a conocer cada uno de los elementos y definiciones fundamentales para comprender y analizar de una manera simple y evidente los diferentes factores y condicionantes a los que se enfrentan los estudiantes que tuvieron que migrar, al igual que el impacto de dicha situación sobre cada una de las familias a las que pertenecen dichas personas.

La investigación se enmarcó dentro un campo de estudio de fácil comprensión y fácil reproducción de técnicas metodológicas y procedimientos, se estudiaron diferentes muestras y una estructura de trabajo que abarca la recopilación de información y su contraste con las investigaciones realizadas. La investigación ratifico y comprobó la importancia que tienen los diferentes factores como, el ambiente social, la psicología del estudiante, el nivel de adaptación, sobre la capacidad y desempeño estudiantil de los mismos Castles (2010).

Este trabajo está relacionado con la investigación en curso ya que nos da a conocer posibles síntomas y factores entre los que se desenvuelven los estudiantes de la carrera de Ingeniería Mecánica de la ESPOCH procedentes de otras provincias y o países.

Dicha actividad se encuentra respaldada por el artículo séptimo del capítulo I De los Derechos Humanos y sus Garantías el cual enuncia lo siguiente:

"Las universidades y las demás instituciones de educación superior a las que la ley otorgue autonomía, tendrán la facultad y la responsabilidad de gobernarse a sí mismas; realizarán sus fines de educar, investigar y difundir la cultura de acuerdo con los principios de este artículo, respetando la libertad de cátedra e investigación y de libre examen y discusión de las ideas; determinarán sus planes y programas; fijarán los términos de ingreso, 
promoción y permanencia de su personal académico; y administrarán su patrimonio. Las relaciones laborales, tanto del personal académico como del administrativo, se normarán por el apartado A del artículo 123 de esta Constitución, en los términos y con las modalidades que establezca la Ley de Trabajo conforme a las características propias de un trabajo especial, de manera que concuerden con la autonomía, la libertad de cátedra e investigación y los fines de las instituciones a que esta fracción se refiere". El presente artículo, secunda la labor de la universidad relacionada al enfoque de los estudiantes en el ámbito investigativo.

"A través de mi experiencia con Piaget de trabajar con un alumno a la vez y tratar de descubrir, desde muy cerca, qué era lo que realmente pasaba por la mente de aquel niño, yo había adquirido una base formidable para despertar mi sensibilidad hacia los alumnos. Creo que una cierta dosis de este tipo de conocimientos y trabajo sería igualmente útil para todos los docentes" (Duckworth, 1999). Esta referencia apoya el hecho de que en el proceso de enseñanza aprendizaje interviene de manera determinante el profesor, este personaje con múltiples historias e influencias psicológicas y sociales que dirige su accionar frente a los alumnos.

En caso de presentarse problemas como las bajas calificaciones, algunos estudiantes se retiran de la carrera. Esto es una muestra de que el campo educativo o formador del joven va de la mano con su capacidad para enfrentarse en el mundo laboral, pero más importante aún su cabida para enfrentar problemas de la vida cotidiana asumiendo con responsabilidad las cosas que le puedan pasar en el transcurso de su vida estudiantil (Levitt \& Glick Schiller, 2004). Se puede afirmar igualmente que todos los docentes poseen bases empíricas y experimentales sobre el proceso de enseñanzaaprendizaje, sin embargo, no es para nada extraño que se sorprendan al observar alguna evaluación por parte de sus estudiantes y se pregunten: “¿Cómo sucedió esto? ¡Si yo lo expliqué muy bien!”

Es necesario cuestionarse qué tipo de educador se desea ser. Sin duda alguna hay quienes, a lo largo de su desempeño, se limitan a presentarse, pasar asistencia y -de una u otra manera-, tratar de "cubrir el programa". Es también sabido que existen aquellos que instruyen a sus pupilos con las mismas prácticas pedagógicas con las que ellos fueron formados, y algunos que imparten sus clases con base en lo que les ha funcionado y lo siguen aplicando en otros contextos, sin ningún ajuste. Casi todas las personas, seguramente, han recibido este tipo de "enseñanza" en algún momento de su vida.

\section{Conclusiones}

Se identificaron los principales factores que afectan el rendimiento académico de los estudiantes migrantes, siendo la adaptación el principal factor. A muchos estudiantes les cuesta mucho responsabilizarse en sus 
estudios y en su vida personal, y también les resulta difícil vivir sin compañía de sus seres queridos.

El factor psicológico es el fundamental particípe en el rendimiento estudiantil, pues un mal estado mental conlleva a un mal estado físico ocasionando un deterioro total del estudiante y un rendimiento bajo.

\section{References:}

1. Castles Stephen (2010). Comprendiendo la migración global: Una perspectiva desde la transformación social. Relaciones Internacionales. (14), 141-169. Obtenido de https://repositorio.uam.es/handle/10486/678145

2. Cristy Fontes Abreu (2016). sifp.psico.edu.uy. Obtenido de https://sifp.psico.edu.uy/sites/default/files/Trabajos\%20finales/\%20A rchivos/tfg_cristy_fontes.pdf

3. Gómez, R.R. (6 de 2005). scielo.org. Obtenido de http://www.scielo.org.mx/scielo.php?script=sci_arttext\&pid=S14057 4252005000200009

4. Levitt, P. \& Glick Schiller, N. (2004). Perspectivas internacionales sobre migración: conceptuar la simultaneidad. Migración y Desarrollo, (3), 60-91. Obtenido de https://www.redalyc.org/html/660/66000305/

5. Núñez, D., Chavarría, V. \& Molina, J. (2011). Influencia De Los Factores Socioeconómicos En La Deserción Estudiantil De La Carrera De Ciencias Sociales. Ciencia E Interculturalidad,6(1), 72-84. Obtenido de https://doi.org/10.5377/rci.v6i1.282. 